This is an Accepted Manuscript of an article published by Taylor \& Francis in Cultura y Educacion on 2019 Mar 18, available online:

http://www.tandfonline.com/10.1080/11356405.2019.1597440

\title{
Validación de un Instrumento de Autopercepción de Competencia Mediática para Docentes en Formación Validation of a self-perceived media competence instrument for pre-service teachers
}

\author{
Julio-César Mateus a; Wilson Hernández-Breña ${ }^{b}$ y Mònica Figueras-Maz ${ }^{a}$ \\ ${ }^{a}$ Universitat Pompeu Fabra; ${ }^{\text {b }}$ Universidad de Lima
}

\begin{abstract}
Resumen: El desarrollo de la competencia mediática en la escuela es clave para garantizar el acceso a derechos humanos fundamentales vinculados con la expresión y la información. Bajo esta premisa, la formación inicial de los docentes en esta materia resulta fundamental para el desarrollo de esta competencia mediática en la ciudadanía. Con la finalidad de aportar un instrumento de diagnóstico útil para orientar a formadores de docentes y decisores de política educativa, diseñamos un cuestionario de autopercepción basado en el constructo teórico de competencia mediática propuesto por Ferrés y Piscitelli (2012), que aborda seis dimensiones. El cuestionario fue aplicado a una muestra de estudiantes de educación peruanos $(N=501)$. Por un lado, los resultados confirman la coherencia del constructo y la validez y confiabilidad del instrumento. Por el otro, nos permiten una actualización teórica del modo en que este constructo se interpreta y aplica, confirmando la multidimensionalidad de la competencia mediática. Finalmente, discutimos a partir de nueva evidencia empírica, el estatuto integral, transversal y ecológico de la competencia mediática.
\end{abstract}

Palabras clave: educación mediática; competencia mediática; cuestionario ad-hoc; validación psicométrica, formación inicial docente

\begin{abstract}
The development of media competence in schools is key to guaranteeing access to fundamental human rights linked to expression and information. Under this premise, the initial training of teachers in this area is essential for the development of this media competence in citizenship. In order to provide a useful diagnostic tool to guide teacher educators and educational policy makers, we designed a self-perception questionnaire based on the theoretical construct of media competence proposed by Ferrés and Piscitelli (2012), which addresses six dimensions. The questionnaire was applied to a sample of Peruvian students of education $(N=$ 501). On the one hand, the results confirm the coherence of the construct and the validity and reliability of the instrument. On the other, they allow us to propose a theoretical update of the way in which this construct is interpreted and applied. Thus, media competence is defined by its multidimensionality. Finally, we discuss from new empirical evidence, the integral, transversal
\end{abstract}


and ecological statute of the media competence.

Keywords: media education; media competence; ad-hoc questionnaire; psychometric validation, teacher initial training

\section{Introducción}

La educación mediática es considerada como un derecho humano porque permite desarrollar una competencia clave para los ciudadanos de este siglo (UNESCO, 2011). Desde esta perspectiva, diversos organismos mundiales promueven su integración efectiva en la formación inicial docente. La aparición en 2011 del Curriculum para Profesores publicado por UNESCO supuso un hito, al ser el primer documento global con definiciones y estándares para la capacitación docente en este campo. Desde entonces, el interés por la formación docente y la discusión sobre cómo se deben integrar los medios a sus planes de estudio ha sido creciente (Andrada, 2015; Pérez-Tornero \& Tayie, 2012; Redecker, 2017).

A pesar de lo anterior, queda mucho camino por recorrer para que la competencia mediática forme parte permanente de la preparación de futuros profesores. Del mismo modo, para que su integración curricular no se limite al aspecto tecnológico, omitiendo otras dimensiones relevantes (Ferrés \& Masanet, 2015; Gutiérrez \& Tyner, 2012; Pérez Escoda \& Rodríguez Conde, 2016). Juega en su contra el hecho de que la competencia mediática tenga aún un nivel de estandarización muy bajo y que el énfasis de cada país o región sea variable, de modo que en algunos prime lo digital, en otros lo informacional, y en otros lo multimedial. Al no existir un consenso en su definición, en su progresión ni en cómo se integra curricularmente, tampoco existe una única forma de evaluarla, lo que dificulta su promoción y crecimiento.

Algunos países iberoamericanos han dado pasos importantes para la definición de políticas curriculares y estándares competenciales en materia de Tecnologías de Información y Comunicación (TIC). Esto resulta importante porque permite saber lo que se espera de los docentes en términos de su formación y práctica profesional, así como identificar sus necesidades específicas (Fernández de la Iglesia, Fernández Morante \& Cebreiro López, 2016, p. 137). Sin embargo, no existen instrumentos de diagnóstico para evaluar el grado de competencia mediática durante la formación docente.

En ese contexto, este artículo puede resultar de especial relevancia para los responsables de diseñar políticas educativas o tomar decisiones en el ámbito de la formación inicial docente. Creemos que la formación inicial docente se convierte en una barrera que entrampa el desarrollo de capacidades mediáticas en la escuela cuando no considera la educación mediática como un componente fundamental. Así nuestro trabajo plantea dos objetivos: por un lado, validar un instrumento original, elaborado a partir del constructo teórico multidimensional propuesto por Ferrés y Piscitelli (2012) y, por el otro, proponer una actualización teórica del modo en que este constructo se interpreta y aplica, tomando como base los resultados empíricos obtenidos.

\section{La competencia mediática}

Las diversas conceptualizaciones de la competencia mediática han generado la creación de 
variados instrumentos que evalúan su grado de desarrollo, algunos de discutible validez. Potter y Thai (2016) hallaron, en una revisión sistematizada de 88 estudios, que al menos la cuarta parte de ellos no ofrecen ninguna definición conceptual precisa, mientras que en el resto de casos se presentan definiciones propias que no siempre miden lo que pretenden medir o lo hacen de una manera inconsistente. Esto ocurre cuando no se demuestra de manera concreta la relación entre la capacidad evaluada y los indicadores específicos diseñados para obtener esa medida, lo que supone un problema de validez de contenido. Como salida, estos autores proponen buscar la máxima transparencia posible utilizando definiciones previamente validadas y no sacrificar la teoría priorizando el pragmatismo.

Para nuestro estudio partimos de la definición propuesta por Joan Ferrés y Alejandro Piscitelli (2012) por considerar que integra dimensiones usualmente fragmentadas en otras definiciones y por haber sido ampliamente utilizada en el ámbito Iberoamericano. Asimismo, su propuesta fue validada por 50 reconocidos expertos internacionales y ofrece definiciones e indicadores precisos, lo que nos permite mitigar el riesgo de falta de transparencia y validez criticada por Potter y Thai.

Para Ferrés y Piscitelli (2012), la competencia mediática "permite interaccionar de manera crítica con mensajes (mediáticos) producidos por los demás, y siendo capaz de producir y de diseminar mensajes propios" (pp. 77-78). Esta interacción crítica exige el desarrollo de una serie de conocimientos, habilidades y actitudes. Esta competencia la organizan en seis dimensiones, sintetizadas en la Tabla 1: (i) lenguajes, (ii) tecnología, (iii) procesos de interacción, (iv) procesos de producción y difusión, (v) ideología y valores, y (vi) dimensión estética. Cada dimensión competencial presenta capacidades correspondientes a los ámbitos de la producción de mensajes propios y de la interacción con mensajes ajenos. Para los autores, tanto las dimensiones como los indicadores han de ser genéricos y flexibles, con el fin de ser adaptados "a cada situación educativa concreta, en función de la edad y del nivel cultural de las personas con las que se ha de trabajar" (p. 77).

Tabla 1. Resumen de indicadores de la competencia mediática

\begin{tabular}{|c|c|}
\hline Dimensión & Definición operacional \\
\hline Lenguaje & $\begin{array}{l}\text { Conocimiento de los códigos, capacidad para utilizarlos y para analizar mensajes } \\
\text { escritos y audiovisuales desde la perspectiva del sentido y el significado, de las } \\
\text { estructuras narrativas y de las categorías y géneros. }\end{array}$ \\
\hline Tecnología & $\begin{array}{l}\text { Conocimiento y capacidad de utilización de las herramientas que hacen posible la } \\
\text { comunicación escrita y audiovisual para entender cómo se elaboran los mensajes. } \\
\text { Comprensión del papel que desempeñan las TIC en la sociedad. }\end{array}$ \\
\hline $\begin{array}{l}\text { Procesos de } \\
\text { interacción }\end{array}$ & $\begin{array}{l}\text { Capacidad de valorar, seleccionar, revisar y autoevaluar la propia dieta mediática. } \\
\text { Capacidad de valorar críticamente los elementos cognitivos, racionales, emocionales } \\
\text { y contextuales que intervienen en el intercambio de mensajes. }\end{array}$ \\
\hline $\begin{array}{l}\text { Procesos de } \\
\text { producción y } \\
\text { difusión }\end{array}$ & $\begin{array}{l}\text { Conocimiento de las funciones y tareas de los agentes de producción, las fases de los } \\
\text { procesos de producción y difusión y los códigos de regulación. Capacidad para } \\
\text { elaborar, seleccionar, compartir y diseminar mensajes mediáticos. }\end{array}$ \\
\hline Ideología & Capacidad de lectura comprensiva y crítica, de análisis crítico y actitud de selección \\
\hline
\end{tabular}


de los mensajes mediáticos, en cuanto representaciones de la realidad.

Estética Capacidad de analizar y de valorar los mensajes audiovisuales desde el punto de vista de la innovación formal y temática y la educación del sentido estético.

Nota: Adaptado de Ferrés y Piscitelli (2012)

Los estudios que se desprenden del desarrollo teórico de Ferrés y Piscitelli parten de la premisa que las dimensiones se pueden medir por separado. Según concluyen diversos trabajos, se da mayor atención a la dimensión tecnológica, limitada a la operación y uso de TIC, en detrimento de otras dimensiones como la estética o la ideológica. Este sesgo se observa de forma sostenida en la producción académica (Andrada, 2015), en los currículos escolares (Medina, Briones \& Hernández, 2017), en los planes de formación inicial docente (López, \& Aguaded, 2015), en las competencias de los docentes en formación (Tiede \& Grafe, 2016) y en ejercicio profesional (Rivera-Rogel, Zuluaga-Arias, Montoya, Romero-Rodríguez \& Aguaded, 2017).

Tenemos, entonces, que las diversas dimensiones de la educación mediática aparecen parcialmente y de forma descompensada en los currículos de las carreras de educación y que su aplicación responde más al interés personal de los docentes o su formación autodidacta antes que al desarrollo de proyectos o políticas institucionales que la promuevan (Ferrés \& Masanet, 2015). Como demuestra una reciente investigación, cuando se mide la competencia mediática de manera multidimensional - como plantean Ferrés y Piscitelli- los alumnos de titulaciones de educación de diez provincias españolas muestran niveles medios y bajos, pero sobre todo una correlación positiva entre la mayor formación recibida y el mejor desarrollo competencial alcanzado, lo que confirma la importancia de la preparación inicial (Ramírez-García \& González-Fernández, 2016).

Finalmente, en cuanto al carácter autoperceptivo de nuestro instrumento, es interesante señalar que la teoría cognitiva social, desarrollada por autores como Albert Bandura (1977), se basa en la premisa de que los seres humanos desarrollamos de forma autónoma las capacidades de regularnos, reflexionar y organizarnos. En este sentido, el que nos consideremos capaces para el desarrollo de tal o cual tarea, se correlaciona de modo favorable con en el logro de las mismas; por el contrario, si nos consideramos poco capaces, esto afecta negativamente nuestro aprendizaje. En el campo docente se sabe que, además de conocimientos y habilidades, los docentes deben creerse capaces de lograr buenos resultados, por lo que el concepto de autoeficacia docente adquiere un valor especial en la investigación educativa (Mahler, Großschedl \& Harms, 2017).

Es obvio que este planteamiento ofrece limitaciones (Potter \& Thai, 2016), pero la finalidad diagnóstica del instrumento que proponemos en este trabajo puede matizarlas en tanto que sus resultados permitirán mejorar o resolver problemas detectados en el proceso formativo de los docentes, más que demostrar sus capacidades concretas. Por lo tanto, indagar en la autoeficacia competencial de los futuros profesores resulta decisivo, más aún cuando la literatura especializada la considera un predictor de disposición y motivación para su práctica pedagógica (Erdem \& Demirel, 2007; Hatlevik, 2016). 


\section{Método}

\section{Instrumento}

Para cumplir con el objetivo de validar un instrumento que responda a nuestra necesidad de medir la autopercepción de competencia mediática, diseñamos un cuestionario ad-hoc que denominamos «Cuestionario de Autopercepción sobre Competencia Mediática para Docentes en Formación».

El cuestionario tiene 30 ítems que corresponden a las seis dimensiones competenciales propuestas por Joan Ferrés y Alejandro Piscitelli (2012). Asimismo, tomamos como referencia para la construcción de los ítems diversos estudios realizados en Iberoamérica basados en ese constructo (Fernández-Planells \& Figueras-Maz, 2012; Gozálvez, González \& Caldeiro, 2014; López, \& Aguaded, 2015; Ramírez \& González, 2016; Rivera-Rogel et al., 2017). Luego de la revisión de literatura científica, creamos estos 30 ítems, cinco para cada dimensión (ver Tabla 2).

En forma previa a la validación psicométrica, el instrumento fue sometido a la evaluación de cinco jueces independientes expertos en educación mediática de España y Latinoamérica (entre ellos, uno de los dos autores del constructo teórico). En el instrumento, cada ítem ofrece una escala de Likert de cinco grados ( $1=$ muy en desacuerdo, $2=$ en desacuerdo, $3=$ indeciso, 4=de acuerdo, y $5=$ muy de acuerdo) para que los participantes valoren sus capacidades percibidas. Los ítems expresan situaciones concretas vinculadas con la educación mediática aplicada a la práctica docente.

Tabla 2.

Dimensiones competenciales e items del cuestionario

\begin{tabular}{lc}
\hline Dimensión & Ítems del Cuestionario \\
\hline $\begin{array}{l}\text { 1. Respecto a los } \\
\text { LENGUAJES de los } \\
\text { medios. Soy capaz } \\
\text { de... }\end{array}$ & $\begin{array}{c}\text { 1.1 Analizar la connotación de cualquier plano y ángulo en una imagen } \\
\text { Utilizar materiales didácticos procedentes de los medios explotando sus } \\
\text { propios códigos } \\
\text { 1.3 Elaborar una rúbrica para evaluar un producto mediático hecho por los } \\
\text { alumnos (ej. un blog o un vídeo) } \\
\text { 1.4 Producir un video a partir de fragmentos de otros videos } \\
\text { 1.5 Distinguir la información de la opinión en una noticia periodística }\end{array}$ \\
\hline $\begin{array}{l}\text { 2. Respecto a la } \\
\text { TECNOLOGÍA de } \\
\text { los medios. Soy } \\
\text { capaz de... }\end{array}$ & $\begin{array}{c}\text { 2.1 Explicar los efectos de los medios en las personas a partir de evidencia } \\
\text { cientíca } \\
\text { fraudulentos, software malicioso, etc.) }\end{array}$ \\
& $\begin{array}{c}\text { 2.3 Producir una plataforma web con distintos tipos de texto (audiovisuales, } \\
\text { hipertextuales, etc.) }\end{array}$ \\
& $\begin{array}{c}\text { 2.4 Resolver sin ayuda de terceros los problemas técnicos de los medios que } \\
\text { utilizo }\end{array}$ \\
& 2.5 Aprovechar diversas tecnologías de acuerdo con mis objetivos didácticos \\
\hline 3. Respecto a los & 3.1 Explicar por qué consumo ciertos contenidos mediáticos, aunque sepa que \\
\hline
\end{tabular}




\begin{tabular}{|c|c|}
\hline PROCESOS DE & no me educan \\
\hline \multirow{4}{*}{$\begin{array}{l}\text { INTERACCIÓN } \\
\text { con los medios. } \\
\text { Soy capaz de... }\end{array}$} & 3.2 Explicar el modelo de negocio de un medio de comunicación \\
\hline & 3.3 Encontrar de manera eficiente la información que requiero en Internet \\
\hline & $\begin{array}{l}\text { 3.4 Producir un texto (impreso o visual) de forma colaborativa utilizando } \\
\text { herramientas en línea }\end{array}$ \\
\hline & $\begin{array}{l}\text { 3.5 Presentar una queja formal por algún contenido mediático que considere } \\
\text { ofensivo ante las instancias correspondientes }\end{array}$ \\
\hline \multirow{5}{*}{$\begin{array}{l}\text { 4. Respecto a la } \\
\text { ESTÉTICA de los } \\
\text { medios. Soy capaz } \\
\text { de... }\end{array}$} & $\begin{array}{l}\text { 4.1 Reconocer una producción audiovisual formalmente deficiente (por la } \\
\text { calidad de imagen, sonido o montaje) }\end{array}$ \\
\hline & $\begin{array}{l}\text { 4.2 Diseñar una presentación multimedia con estándares estéticos } \\
\text { (armonía, composición, etc.) }\end{array}$ \\
\hline & 4.3 Identificar encuadres incorrectos en una fotografía \\
\hline & $\begin{array}{l}\text { 4.4 Reconocer influencias mutuas entre producciones culturales y otras } \\
\text { manifestaciones artísticas }\end{array}$ \\
\hline & $\begin{array}{l}\text { 4.5 Producir determinadas emociones a partir de la elaboración de un } \\
\text { contenido }\end{array}$ \\
\hline \multirow{5}{*}{$\begin{array}{l}\text { 5. Respecto a la } \\
\text { IDEOLOGÍA Y } \\
\text { VALORES de los } \\
\text { medios. Soy capaz } \\
\text { de... }\end{array}$} & $\begin{array}{l}\text { 5.1 Explicar aspectos legales básicos vigentes en mi país relacionados con los } \\
\text { medios }\end{array}$ \\
\hline & $\begin{array}{l}\text { 5.2 Advertir riesgos latentes en la red vinculados a la privacidad o la identidad } \\
\text { digital }\end{array}$ \\
\hline & $\begin{array}{l}\text { 5.3 Reconocer los procesos de identificación emocional con personajes o } \\
\text { historias y sus efectos }\end{array}$ \\
\hline & 5.4 Contrastar la fiabilidad de la información que obtengo en medios digitales \\
\hline & $\begin{array}{l}\text { 5.5 Advertir estereotipos en una publicidad y explicar sus efectos a partir de } \\
\text { evidencia científica }\end{array}$ \\
\hline \multirow{5}{*}{$\begin{array}{l}\text { 6. Respecto a la } \\
\text { PRODUCCIÓN Y } \\
\text { DIFUSIÓN de los } \\
\text { medios. Soy capaz } \\
\text { de... }\end{array}$} & $\begin{array}{l}\text { 6.1 Definir qué es un medio de comunicación público y decir cuáles existen } \\
\text { en el país. }\end{array}$ \\
\hline & 6.2 Enumerar al menos ocho cargos responsables de elaborar una película \\
\hline & $\begin{array}{l}\text { 6.3 Advertir conflictos que se puedan crear entre los intereses de los } \\
\text { propietarios de un medio, los que lo financian y la información que } \\
\text { emite }\end{array}$ \\
\hline & 6.4 Elaborar un guion audiovisual con estándares técnicos \\
\hline & 6.5 Diseñar un Entorno Personal de Aprendizaje (PLE) eficiente y confiable \\
\hline
\end{tabular}

\section{Participantes}

La muestra final de participantes en el cuestionario estuvo compuesta por 501 estudiantes de educación de cuatro instituciones de Lima, tres universidades y un instituto pedagógico que también habilita para el ejercicio profesional. Diversas características adicionales aseguran la heterogeneidad de la muestra empleada. Del total, el 86\% son mujeres, proporción que refleja bastante bien la proporción de mujeres y hombres que siguen la carrera de educación en el Perú. La mayoría nació en la capital (64\%) y la edad promedio fue de 21 años (38\% de la muestra era menor de esa edad), con una desviación estándar baja (2.46). Las especialidades estuvieron bastante bien representadas: el 30\% eran de educación inicial, el $41 \%$ de primaria y el 29\% de secundaria. La mayoría de los estudiantes cursaba el segundo año de carrera (40\%) mientras que de tercer año participaron el 13\% y de los dos años siguientes, las proporciones fueron algo 
mayores $(25 \% \text { y } 22 \% \text {, respectivamente })^{1}$. Finalmente, poco más de la mitad de la muestra estudiaba en una universidad (58\%) y el resto en un instituto pedagógico (42\%).

La selección de los centros de estudios fue no probabilística por cuotas y respondió a los siguientes criterios: que fueran (i) centros educativos de reconocida calidad ${ }^{2}$ y (ii) que estuvieran dispuestos a participar del estudio de forma institucional. Sus equipos directivos mostraron disposición e interés en llevar a cabo este diagnóstico.

\section{Procedimiento}

El instrumento fue aplicado en coordinación con las instituciones participantes entre el 15 de setiembre y 15 de octubre de 2017. Los estudiantes fueron informados del propósito de la investigación y participaron de manera voluntaria. El cuestionario fue autoadministrado y la duración promedio de respuesta fue de 20 minutos. La tasa de preguntas sin contestar fue baja (entre $0.8 \%$ y $2.99 \%$ ) y no representa riesgo de error sistemático en los resultados. Los datos estadísticos fueron procesados con el software Stata 14.

La consistencia interna del instrumento fue evaluada mediante el estadístico Alpha de Cronbach y el Índice de Homogeneidad (correlación ítem-total), lo cual permitió evaluar la variación de las varianzas al eliminar cada ítem. Esta evaluación global fue complementada con la validez para cada una de las dimensiones del instrumento. Como la confiabilidad lograda fue importante (0.8139), no fue necesario realizar procedimientos adicionales para identificar cuántos ítems adicionales habría que agregar para obtener un nivel de confiabilidad deseado (mayor a 0.80).

Adicionalmente, se estimaron las medidas de Kaiser-Meyer-Olkin (KMO) para evaluar la proporción de la varianza que obedece a los factores subyacentes evaluados. Esta medida se complementó con la prueba de esfericidad de Barlett a fin de evaluar la relación entre los ítems y dar paso a pruebas que detecten una estructura mayor en la data. La validez del constructo fue analizada mediante Análisis Factorial Confirmatorio, habida cuenta de la existencia de modelos previos. Diversos indicadores fueron empleados para evaluar la bondad de ajuste (Chi2, Root Mean Squared Error of Approximation, Standardized Root Mean Squared Residual, Comparative Fit Index y Non-Normed Fit Index). Finalmente, se realizaron distintas pruebas de validez discriminante, para lo que se contrastó el puntaje total del cuestionario entre el primer y último tercil (t-test). La data obtenida fue procesada en el software Stata versión 14.

\footnotetext{
${ }^{1}$ El grado universitario de educación en el Perú tiene cinco años de duración.

${ }^{2}$ Para cumplir con este criterio elegimos centros reconocidos por la Superintendencia Nacional de Educación Superior (Sunedu), en el caso de las universidades, y por el Sistema Nacional de Evaluación, Acreditación y Certificación de la Calidad Educativa (Sineace), en el caso del instituto pedagógico.
} 


\section{Resultados}

\section{Análisis factorial}

Para determinar la validez del instrumento, realizamos en primer lugar un Análisis Factorial Exploratorio. Producto de éste, se identificó que los 30 ítems se agrupan en torno a sólo cuatro dimensiones. Pero sólo tres de ellas tienen Eigenvalues mayores a uno $(10,88 ; 1,39 ; 1,12)$. Además, la primera dimensión destaca por encima de las otras dos. La segunda y la tercera dimensión tienen pocos ítems, mezclan ítems de distintas dimensiones teóricas y presentan comunalidades negativas y más bajas que su contribución a la primera dimensión. En resumen, esto afianza la conclusión de una única dimensión detrás del constructo evaluado. El Análisis Factorial Confirmatorio, con seis dimensiones, arroja evidentemente el mismo resultado. En suma, es más coherente concluir que los 30 ítems de nuestro instrumento contribuyen a una única dimensión, cuyo Eigenvalue, además, es bastante alto (10.88) que el resto $(<1.39)$. Empíricamente, este resultado discute aquellos trabajos que, basados en el desarrollo teórico de Ferrés y Piscitelli (2012), asumen que la competencia mediática es la suma de seis dimensiones independientes que pueden evaluarse de modo parcial.

Teniendo en cuenta lo anterior, se retuvieron los 30 ítems para el análisis de la validez interna. Ningún ítem presentó problemas de asimetría, curtosis ni valores promedios muy altos (ceilling effect). Situaciones de este tipo hubieran introducido problemas de variabilidad en los datos.

A fin de evaluar la consistencia interna del instrumento se recurrió al Alpha de Cronbach. El alto valor obtenido (0.9416) se ubica en un rango ideal (Kline, 2000), lo cual señala dos aspectos. Primero, que la varianza entre los ítems está fuertemente asociada y que, por tanto, reflejan en forma satisfactoria la medición de la competencia mediática como un constructo latente. Segundo, la diversidad de dimensiones competenciales recogidas en los ítems asegura una correlación no artificial entre ellos.

En apoyo a la validación interna, el índice de homogeneidad (Correlación ítem-test) mostró correlaciones homogéneas e importantes (entre 0.43 y 0.73 ), aspecto que asegura que la medición de cada ítem desde lo empírico tiene conexión con el instrumento en su conjunto y con el planteamiento teórico del mismo. La factibilidad de realizar el Análisis Factorial estuvo previamente refrendada por el KMO, cuyo valor (0.941) se ubicó dentro de un rango bastante satisfactorio, y el test de esfericidad de Barlett ( $p$-value $<0.001$ ). Mientras que lo primero prueba la adecuación de la muestra, lo segundo descarta que las variables no estén correlacionadas.

Los resultados del Análisis Factorial se presentan en la Tabla 3. En todos los ítems, las comunalidades (dimensión 1) tuvieron un valor mayor a 0.40 , lo que significa que una mayor parte de la varianza de la autopercepción de competencia mediática puede ser explicada por los ítems evaluados. Además, esta relación estadística es fuerte y asegura la producción de buenos estimadores cuando la muestra es de 200 observaciones (Fabrigar, Wegener, MacCallum \& Strahan, 1999). La muestra empleada en este estudio fue de más del doble de este estándar. Como resultado, nuestro instrumento muestra propiedades adecuadas para el análisis desarrollado.

Tabla 3.

Análisis Factorial y tests de correlación

-POSTPRINT- 


\begin{tabular}{|c|c|c|c|c|c|}
\hline Item & $\begin{array}{c}\text { Comunalidades } \\
\text { del Factor } 1\end{array}$ & $\begin{array}{c}\text { Correlación } \\
\text { ítem-test }\end{array}$ & $\begin{array}{c}\text { Correlación } \\
\text { ítem-resto }\end{array}$ & $\begin{array}{c}\text { Covarianza } \\
\text { inter-ítem } \\
\text { promedio }\end{array}$ & Alpha \\
\hline com_len1 & 0.58 & 0.5842 & 0.5488 & 0.3482 & 0.9400 \\
\hline com_len2 & 0.53 & 0.5474 & 0.5071 & 0.3494 & 0.9403 \\
\hline com_len3 & 0.51 & 0.5333 & 0.4911 & 0.3486 & 0.9406 \\
\hline com_len4 & 0.54 & 0.5379 & 0.4958 & 0.3483 & 0.9405 \\
\hline com_len5 & 0.52 & 0.5219 & 0.4854 & 0.3518 & 0.9406 \\
\hline com_tec1 & 0.57 & 0.5915 & 0.5562 & 0.3479 & 0.9399 \\
\hline com_tec 2 & 0.54 & 0.5677 & 0.5274 & 0.3473 & 0.9402 \\
\hline com_tec3 & 0.58 & 0.6108 & 0.5691 & 0.3427 & 0.9399 \\
\hline com_tec4 & 0.59 & 0.5973 & 0.5560 & 0.3443 & 0.9398 \\
\hline com_tec5 & 0.60 & 0.6096 & 0.5770 & 0.3482 & 0.9397 \\
\hline com_int1 & 0.49 & 0.4895 & 0.4493 & 0.3528 & 0.9409 \\
\hline com_int2 & 0.60 & 0.6328 & 0.5985 & 0.3449 & 0.9395 \\
\hline com_int3 & 0.48 & 0.4831 & 0.4511 & 0.3559 & 0.9409 \\
\hline com_int4 & 0.45 & 0.4366 & 0.3934 & 0.3554 & 0.9413 \\
\hline com_int5 & 0.67 & 0.6778 & 0.6417 & 0.3394 & 0.9389 \\
\hline com_est1 & 0.61 & 0.5995 & 0.5650 & 0.3478 & 0.9398 \\
\hline com_est2 & 0.66 & 0.6397 & 0.6065 & 0.3452 & 0.9394 \\
\hline com_est3 & 0.65 & 0.6495 & 0.6126 & 0.3418 & 0.9393 \\
\hline com_est4 & 0.73 & 0.7310 & 0.7030 & 0.3401 & 0.9384 \\
\hline com_est5 & 0.67 & 0.6733 & 0.6416 & 0.3435 & 0.9390 \\
\hline com_ide1 & 0.65 & 0.6657 & 0.6299 & 0.3405 & 0.9390 \\
\hline com_ide2 & 0.65 & 0.6731 & 0.6402 & 0.3418 & 0.9390 \\
\hline com_ide3 & 0.66 & 0.6734 & 0.6397 & 0.3420 & 0.9390 \\
\hline com_ide4 & 0.63 & 0.6373 & 0.6066 & 0.3468 & 0.9394 \\
\hline com_ide5 & 0.61 & 0.6265 & 0.5899 & 0.3439 & 0.9395 \\
\hline com_pro1 & 0.59 & 0.5924 & 0.5572 & 0.3475 & 0.9399 \\
\hline com_pro2 & 0.61 & 0.6236 & 0.5855 & 0.3432 & 0.9396 \\
\hline com_pro3 & 0.69 & 0.7034 & 0.6710 & 0.3395 & 0.9387 \\
\hline com_pro4 & 0.67 & 0.6948 & 0.6612 & 0.3388 & 0.9387 \\
\hline com_pro5 & 0.62 & 0.6403 & 0.6006 & 0.3407 & 0.9395 \\
\hline Alpha & & & & & 0.9416 \\
\hline
\end{tabular}

\section{Bondad de ajuste}

Un factor importante en este tipo de análisis es evaluar la bondad de ajuste del modelo. Es decir, mide qué tan bien nuestro modelo empírico se ajusta al modelo teórico esperado. El estadístico Root Mean Squared Error of Approximation fue de 0.094, ubicándose muy por debajo del tope máximo permitido que sugieren MacCallum, Browne y Sugawara (1996). Es probable que este resultado esté relacionado por el uso de un modelo parsimonioso (relativamente pocas 
variables) (Schubert, Hagemann, Voss \& Bergmann, 2017). Asimismo, la diferencia estandarizada observada entre la correlación predicha y esperada (Standardized root mean squared residual) fue baja 0.062 y menor al estándar fijado por Hu y Bentler (1999). La bondad de ajuste resulta no fue satisfactoria con el Comparative Fit Index (0.747) y el índice de TuckerLewis (0.729), los que se ubican por debajo de los niveles óptimos de 0.90 y 0.95 . Ambos indicadores evalúan el ajuste entre el modelo evaluado y el hipotetizado empleando el valor chi cuadrado. Es útil tener en cuenta que existen diversas formas de evaluar la bondad de ajuste, las cuales según Mulaik et al. (1989) en general sólo proporcionan la evidencia más fundamental sobre el ajuste del modelo.

\section{Discriminación en la muestra}

Una de las pruebas adicionales importantes en la validación de un instrumento es su capacidad de reflejar distintas intensidades del constructo evaluado. A fin de probarlo, construimos una variable que suma el puntaje individual de cada uno de los 30 ítems. Creamos terciles, cuartiles y quintiles y comparamos las puntuaciones de los grupos extremos. En estos tres casos, se registraron diferencias significativas en el valor individual de cada ítem de tales grupos extremos (p-value siempre menor a 0.01). La prueba de análisis de varianza (ANOVA) corroboró también dichas diferencias en forma significativa, confirmando que el instrumento evaluado tiene la capacidad de diferenciar puntuaciones entre individuos y captar intensidades distintas en la expresión de la autopercepción de competencia mediática.

\section{Correlación entre dimensiones}

Finalmente, como se señaló líneas arriba, el análisis factorial claramente determina desde lo empírico que la competencia mediática es multidimensional, como sugieren Ferrés y Piscitelli (2012), y que no debería ser evaluada aislando cada dimensión. Una prueba adicional de este hallazgo está en la correlación entre estas dimensiones (Tabla 4 y Figura 1).

Tabla 4.

Correlación entre dimensiones

\begin{tabular}{|c|c|c|c|c|c|c|}
\hline & len & tec & int & est & ide & pro \\
\hline len & 1.0000 & & & & & \\
\hline tec & 0.6599 & 1.0000 & & & & \\
\hline int & 0.5518 & 0.5537 & 1.0000 & & & \\
\hline est & 0.5016 & 0.5266 & 0.6435 & 1.0000 & & \\
\hline ide & 0.4521 & 0.5184 & 0.6237 & 0.6596 & 1.0000 & \\
\hline pro & 0.4823 & 0.5219 & 0.5480 & 0.6657 & 0.6898 & 1.0000 \\
\hline
\end{tabular}

Como se aprecia en la Figura 1, la correlación y la forma de las nubes de puntos son bastante similares. Esto ahonda en el hallazgo de que todas las dimensiones forman parte de una misma competencia evaluada desde lo empírico. 


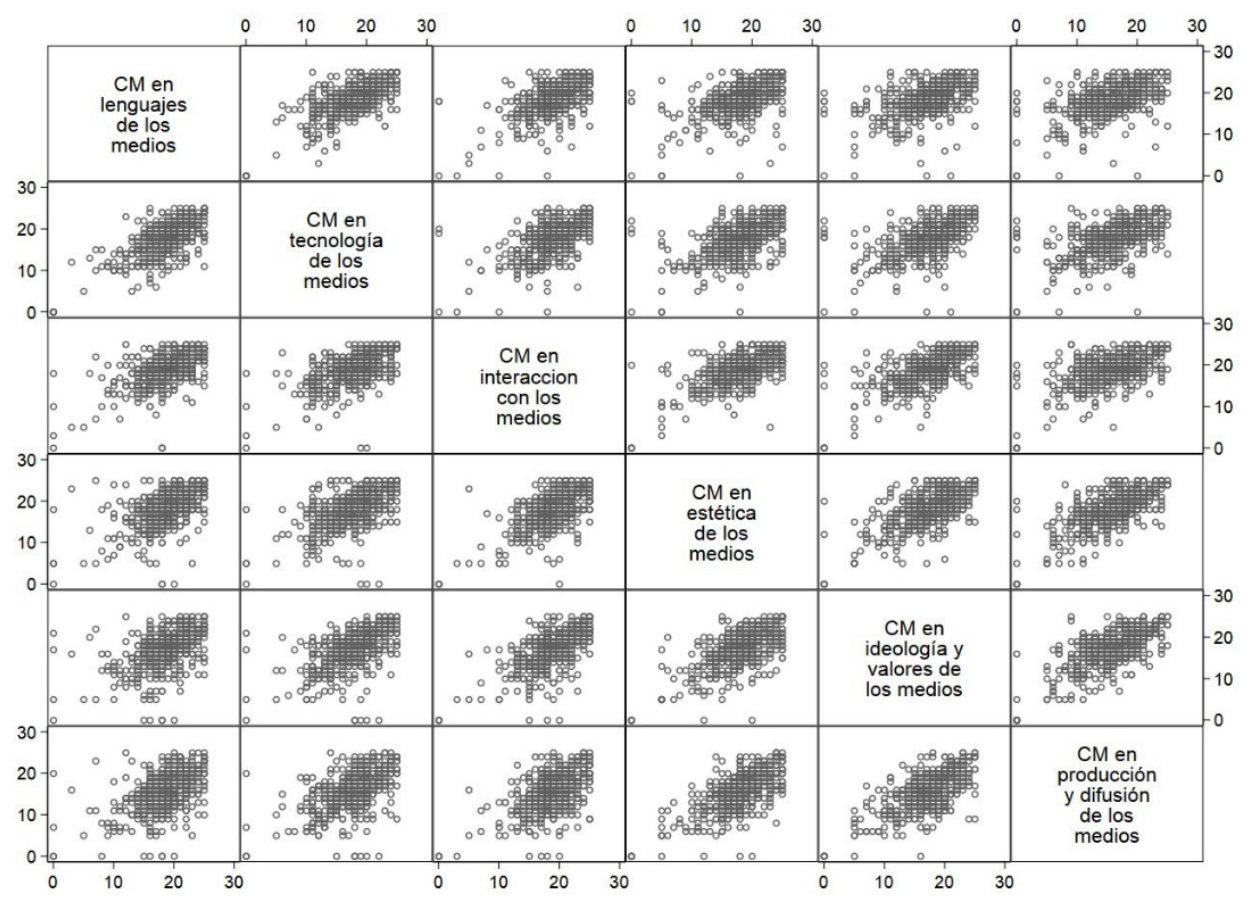

Figura 1. Correlación gráfica entre dimensiones

Como se demuestra, los ítems de cada dimensión funcionan de mejor forma cuando son asociados con aquellos de otras dimensiones. A fin de evaluar este último criterio, se estimó un reporte de modificación de índices para covarianzas. Este análisis sugiere qué covarianzas (correlación entre un índice y otro) deben agregarse al análisis factorial a fin de mejorar su bondad de ajuste. De ser cierto que las dimensiones pueden aislarse, como plantean diversos estudios de competencia mediática, entonces el reporte de modificación de índices debió sugerir introducir covarianzas únicamente entre los ítems de cada dimensión de forma aislada. Sin embargo, el reporte no fue tal. Por el contrario, sugiere la introducción de 167 covarianzas que involucran a la mayoría de los 30 ítems en forma cruzada (es decir, independientemente a la dimensión teórica a la que pertenecen). En suma, y en línea con lo antes señalado, las dimensiones teóricas de la competencia mediática serían más un criterio taxonómico que dimensiones para evaluar un constructo mayor.

Podemos decir, a partir de la evidencia, que este instrumento también resulta útil para obtener una medida de competencia total, integrada por todas las dimensiones que aparecen fuertemente correlacionadas. No son, por lo tanto, compartimentos estancos, sino que se deben entender como un sistema cuyos elementos resultan interdependientes. Esto puede generar, teóricamente, lo que se conoce como una externalidad positiva en la medida en que, si bien las dimensiones no deben evaluarse de forma autónoma, el que se desarrollen de forma parcial también impacta favorablemente al conjunto. 


\section{Discusión y conclusiones}

El nuevo impulso de la educación mediática en el sistema escolar por parte de organizaciones internacionales ha abierto una agenda de investigación importante, en la que se inscribe este trabajo. En este marco, aparecen cada vez más trabajos teóricos y empíricos que se ocupan de caracterizar el consumo de medios o las prácticas mediáticas de los estudiantes, pero no existe todavía suficiente literatura científica que aborde la formación inicial docente. Este artículo vuelve la mirada sobre la preparación de los docentes, que consideramos prioritaria para el desarrollo de la educación mediática en los niños y niñas en la formación de la ciudadanía.

Por otro lado, desde la publicación del constructo de competencia mediática de Ferrés y Piscitelli, el año 2012, se han sucedido diversos estudios que han priorizado unas dimensiones sobre otras o que las han utilizado para comparar el mayor o menor grado de desarrollo competencial de forma particular, y se ha venido sosteniendo que las diversas capacidades pueden medirse de forma aislada.

A diferencia de validaciones previas de escalas de competencia mediática, en este trabajo aplicamos un set completo de técnicas vinculadas al análisis factorial y diversas pruebas adicionales que aportan seguridad sobre su solvencia teórica y empírica. Se trata, por lo tanto, de una propuesta teórica validada empíricamente, lo que actualiza su vigencia y aplicabilidad. Esta herramienta diagnóstica sirve para autoevaluar el grado de competencia mediática en docentes en formación y puede resultar útil para los formadores docentes, los responsables del diseño de planes de estudio y los decisores de política educativa interesados en integrar la educación mediática a la escuela, pues permite recoger la percepción de los involucrados sobre sus propias capacidades mediáticas desde una perspectiva multidimensional, no centrada en lo tecnológico ni conceptualmente sesgada.

Nuestro estudio confirma la coherencia de este constructo teórico, pero al mismo tiempo llama la atención sobre el sentido de evaluar las seis dimensiones que componen la competencia mediática de manera integrada, como advirtieron Potter y Thai (2016) al revisar otros instrumentos de evaluación de esta competencia. El instrumento que hemos utilizado y sometido a validación resulta útil para obtener una medida de competencia total, integrada por las seis dimensiones que la componen porque, como hemos demostrado, aparecen fuertemente correlacionadas. Esto nos permite profundizar en la propuesta teórica original de Ferrés y Piscitelli a partir de tres premisas que consideramos que sostienen la competencia mediática y definen su estatuto integral, transversal y ecológico.

Primero, concluimos que la competencia mediática es integral porque funciona como un sistema: no es posible fragmentar las capacidades de interacción con los medios sino para ofrecer alguna forma mínima de organizarla, porque, en la realidad, el funcionamiento de los medios y nuestra relación con ellos trasciende a los dispositivos y los contenidos que fluyen por ellos. La educación mediática es un asunto de implicación cultural y no puramente tecnológico. Es vital, en esta línea, promover un cambio en el rol docente, desde su formación inicial, para paliar la disonancia digital creada entre las capacidades aprendidas por los estudiantes en el espacio extraescolar y las pocas que son atendidas en el ámbito formal. Existe, como confirma Scolari (2018), una brecha innecesaria que afecta la propia formación docente en un contexto donde los 
jóvenes participan intensamente en entornos digitales y muchos docentes consideran que eso los hace competentes. En ese sentido, como advierte danah boyd (2014), "la retórica del 'nativo digital', lejos de sernos útil, a menudo es una distracción para entender los retos a los que se enfrenta la juventud en un mundo informatizado" (p. 337)

Segundo, decimos que es transversal en la medida en que la competencia mediática atraviesa todos los ámbitos vitales. Los medios son mecanismos de expresión e interacción inherentes a las personas en un contexto de mediatización (Couldry \& Hepp, 2017). Por ejemplo, nos ocupamos ahora de las "fake news" y las "posverdades" sólo desde la capacidad de gestionar la información, cuando, en realidad, atraviesa otras dimensiones competenciales como la de producción (donde importa desde conocer que existen códigos de regulación de los medios hasta saber cómo se viralizan los mensajes) o la de los procesos de interacción (para comprender por qué creemos relatos dudosos cuando cumplen una función emocional). En cuanto a la transversalidad, es oportuno recordar la alteración cultural que las TIC suponen en la condición humana, lo que las convierte no en herramientas sino en fuerzas ambientales que afectan nuestra identidad, nuestra forma de socializar, nuestra concepción de la realidad y nuestra interacción con esa realidad (Floridi, 2015). En ese sentido, la competencia mediática es compleja y tiene implicaciones éticas, estéticas, políticas y económicas que no pueden pasar desapercibidas en la formación de los futuros formadores, ni menos ser consideradas de forma autónoma unas de otras.

Finalmente, decimos que la competencia mediática es ecológica porque es dinámica y está en constante evolución: todo el tiempo irrumpen nuevas interfaces $\mathrm{y}$, con ellos, prácticas de apropiación de esos medios que suelen resultar ajenas a la escuela hasta que son formalizadas por el sistema escolar (Scolari, 2018).

Todo lo anterior hace de la competencia mediática un conjunto de capacidades en continua expansión: iterativas, reticulares e inestables que dependen en gran medida de los avances tecnológicos y que exigen la actualización constante de instrumentos de diagnóstico para saber cómo se están incorporando en la formación docente. En este artículo se presenta una propuesta que se ha comprobado válida, pero será necesario revisarla periódicamente y actualizarla atendiendo a los cambios del ecosistema de medios y los impactos socioculturales y educativos que implica. 


\section{Referencias}

Álvarez, J.F. \& Gisbert, M. (2015). Grado de alfabetización informacional del profesorado de Secundaria en España: Creencias y autopercepciones. Comunicar, 45, 187-194. doi: https://doi.org/10.3916/C45-2015-20

Andrada, P. (2015). Estado de la cuestión de la formación docente en educación en medios. Comunicación y Medios, 32, 83-99. doi: http://dx.doi.org/10.5354/0719-1529.2015.36700

Bandura, A. (1977). Self-efficacy: Toward a unifying theory of behavioral change. Psychological Review, 84, 191-215.

boyd, d. (2014) It's complicated: The Social Lives of Networked Teens. Londres-New Haven: Yale University Press.

Couldry, N. \& Hepp, A. (2017) The mediated construction of reality. Cambridge: Polity.

Erdem, E., \& Demirel, O. (2007). Teacher self-efficacy belief. Social Behavior and Personality, 35, 573-586. doi: https://doi.org/10.2224/sbp.2007.35.5.573

Fabrigar, L., Wegener, D., MacCallum, R., \& Strahan, E. (1999). Evaluating the Use of Exploratory Factor Analysis in Psychological Research. Psychological Methods, 4(3), 272299. doi: http://dx.doi.org/10.1037/1082-989X.4.3.272

Fernández de la Iglesia, J. \& Fernández Morante, M. \& Cebreiro López, B. (2016). Desarrollo de un cuestionario de competencias en tic para profesores de distintos niveles educativos. Pixel-Bit. Revista de Medios y Educación, 48, 135-148. doi: http://dx.doi.org/10.12795/pixelbit.2016.i48.09

Fernández-Planells, A. \& Figueras-Maz, M. (2012) La educación mediática: una asignatura pendiente en las escuelas de Barcelona y Lima. IV Congreso Internacional Latina de Comunicación Social. Universidad de La Laguna. Recuperado de http://www.revistalatinacs.org/12SLCS/2012_actas/064_Fernandez.pdf

Ferrés, J. \& Masanet, M. (2015). La educación mediática en la universidad española. Barcelona: Gedisa.

Ferrés, J. \& Piscitelli, A. (2012). La competencia mediática: propuesta articulada de dimensiones e indicadores. Comunicar, 38, 75-82. doi: https://doi.org/10.3916/C38-2012-02-08

Floridi, L. (Ed.) (2015). The Onlife Manifesto: Being Human in a Hyperconnected Era. Londres: Springer. doi: http://doi.org/10.1007\%2F978-3-319-04093-6

García-Ruiz, Rosa, Gozálvez Pérez, Vicent, \& Aguaded Gómez, J. Ignacio. (2014). La competencia mediática como reto para la educomunicación: instrumentos de evaluación. Cuadernos.info, 35, 15-27. doi: https://dx.doi.org/10.7764/cdi.35.623 
Gozálvez, V., González, N. y Caldeiro, M. C. (2014). La competencia mediática del profesorado: un instrumento para su evaluación. Revista Electrónica de Investigación Educativa, 16(3), 129-146. Recuperado de http://redie.uabc.mx/vol16no3/contenido-gonzalvez-glezcaldeiro.html

Gutiérrez, A. \& Tyner, K. (2012). Educación para los medios, alfabetización mediática y competencia digital. Comunicar, 38, 31-39. doi: https://doi.org/10.3916/C38-2012-02-03

Hatlevik, O.E. (2016). Examining the Relationship between Teachers' Self-Efficacy, their Digital Competence, Strategies to Evaluate Information, and use of ICT at School. Scandinavian Journal of Educational Research, 5(61), 555-567. doi: http://dx.doi.org/10.1080/00313831.2016.1172501

Hu, L. T. \& Bentler, P. M. (1999). Cutoff criteria for fit indexes in covariance structure analysis: Conventional criteria versus new alternatives. Structural equation Modeling, 6(1), 1-55. doi: https://doi.org/10.1080/10705519909540118

López, L. \& Aguaded, M.1. (2015). La docencia sobre alfabetización mediática en las facultades de Educación y Comunicación. Comunicar, 44, 187-195. doi: https://doi.org/10.3916/C44$\underline{2015-20}$

MacCallum, R. C., Browne, M. W., \& Sugawara, H. M. (1996). Power analysis and determination of sample size for covariance structure modeling. Psychological Methods, 1(2), 130-149.

Mahler, D.; Großschedl, J. \& Harms, U. (2017) Opportunities to Learn for Teachers' SelfEfficacy and Enthusiasm. Education Research International, 2017. 1-17. doi: https://doi.org/10.1155/2017/4698371

Medina, F.; Briones, A. J. \& Hernández, E. (2017). Educación en medios y competencia mediática en la educación secundaria en España, Icono 14, 15(1), 42-65. doi: http://dx.doi.org/10.7195/ri14.v15i1.1001

Mulaik, S., James, L., Van Alstine, J., Bennett, N., Lind, S. \& Stilwell, D. (1989). Evaluation of goodness-of-fit indices for structural equation models. Psychological Bulletin, 105(3), 430445 .

Pérez-Tornero, J.M. \& Tayie, S. (2012). La formación de profesores en educación en medios: currículo y experiencias internacionales. Comunicar, 39, 10-14. doi: https://doi.org/10.3916/C39-2012-02-00

Pérez Escoda, A. \& Rodríguez Conde, M. (2016). Evaluación de las competencias digitales autopercibidas del profesorado de Educación Primaria en Castilla y León (España). Revista de Investigación Educativa, 34(2), 399-415. doi: http://dx.doi.org/10.6018/rie.34.2.215121 
Potter, J. \& Thai, C. (2016). Conceptual Challenges in Designing Measures for Media Literacy Studies. International Journal of Media and Information Literacy, 1(1), 27-42. doi: http://dx.doi.org/10.13187/ijmil.2016.1.27

Ramírez-García, A. \& González-Fernández, N. (2016). Competencia mediática del profesorado y del alumnado de educación obligatoria en España. Comunicar, 49, 49-58. doi: https://doi.org/10.3916/C49-2016-05

Ramírez, E., Cañedo, I. \& Clemente, M. (2012). Las actitudes y creencias de los profesores de secundaria sobre el uso de Internet en sus clases. Comunicar, 38, 147-155. https://doi.org/10.3916/C38-2012-03-06

Redecker, C. (2017). European Framework for the Digital Competence of Educators: DigCompEdu. Luxemburgo: Oficina de Publicaciones de la Unión Europea. doi: http://dx.doi.org/10.2760/159770

Rivera-Rogel, D; Zuluaga-Arias, L.; Montoya, N.; Romero-Rodríguez, L. \& Aguaded, I. (2017). Media Competencies for the Citizenship Training of Teachers from Andean America: Colombia and Ecuador. Paidéia (Ribeirão Preto), 27(66), 80-89. doi: https://dx.doi.org/10.1590/1982-43272766201710

Scolari, C.A. (Ed) (2018) Adolescentes, medios de comunicación y culturas colaborativas: aprovechando las competencias transmedia de los jóvenes en el aula. Barcelona: Universitat Pompeu Fabra. Recuperado de https://repositori.upf.edu/handle/10230/34245

Schubert, A., Hagemann, D., Voss, A. \& Bergmann, K. (2017). Evaluating the model fit of diffusion models with the root mean square error of approximation. Journal of Mathematical Psychology, 77, 29-45. doi: https://doi.org/10.1016/j.jmp.2016.08.004

Tiede, J. \& Grafe, S. (2016). Pedagogía mediática en la formación de profesores de Alemania y EEUU. Comunicar, 49, 19-28. doi: https://doi.org/10.3916/C49-2016-02

UNESCO (2011). Alfabetización mediática e informacional. Currículum para profesores. Recuperado de http://unesdoc.unesco.org/images/0021/002160/216099S.pdf 